\title{
Effect of vitamin D deficiency in Korean patients with acute respiratory distress syndrome
}

Sojung Park ${ }^{1}$, Min Gee Lee ${ }^{2}$, Sang-Bum Hong ${ }^{3}$, Chae-Man Lim³, Younsuck Koh ${ }^{3}$ and Jin Won Huh

${ }^{1}$ Department of Pulmonary, Allergy, and Critical Care Medicine, Hallym University Dongtan Sacred Heart Hospital, Hwaseong; Departments of ${ }^{2}$ Internal Medicine and ${ }^{3}$ Pulmonary and Critical Care Medicine, Asan Medical Center, University of Ulsan College of Medicine, Seoul, Korea
Korean J Intern Med 2018;33:1129-1136

https://doi.org/10.3904/kjim.2017.380

In the article "Effect of vitamin D deficiency in Korean patients with acute respiratory distress syndrome" by Park S et al. (Korean J Intern Med 2018;33:11291136) byline mistakenly indicated that Min Gee Lee. There is a spelling mistake of an author's name, and they want to change to Min Gee Lee from Min Gi Lee.

We apologize for any inconvenience that this may have caused. 